\title{
LA CRISIS DEL SECTOR AGROPECUARIO COLOMBIANO: ¿CUÁL ES LA RESPONSABILIDAD DE LAS POLÍTICAS PÚBLICAS?
}

\author{
Por: Elizabeth Rodríguez ${ }^{1}$ - Gloria Lucía Martínez² - Jairo Mora-Delgado ${ }^{3}$
}

\section{RESUMEN}

El sector agropecuario resulta estratégico para la soberanía de un país, ya que es fuente de alimentos y materias primas requeridos por la sociedad para garantizar las necesidades básicas. Este análisis sistemático tiene como objetivo hacer una reflexión de la evolución de las políticas públicas relacionadas con el sector agropecuario. Se revisó documentos de expertos publicados en los últimos treinta años. Se concluye que la política actual del gobierno colombiano ha seguido al pie de la letra los dictámenes del consenso de Washington y ha gobernado con políticas nada convenientes para el desarrollo del sector agropecuario nacional.

Palabras clave: política, neoliberalismo, proteccionismo, agricultura, seguridad alimentaria

Clasificación JEL: Q180.

1. Magister en Estudios y Gestión del Desarrollo. Profesora catedrática, Facultad de Ciencias Humanas y Artes. Universidad del Tolima (Colombia). E-mail: erodriguezh@ut.edu.co

2. Estudiante de Maestría en Desarrollo Rural. Laboratorio de Ecofisiología, Facultad de Medicina Veterinaria y Zootecnia. Universidad del Tolima (Colombia). E-mail: glmartinezr@ut.edu.co

3. PhD en Sistemas de Producción Agrícola. Profesor asociado, Coordinador del Grupo de Investigación en Sistemas Agroforestales Pecuarios, Universidad del Tolima (Colombia). E-mail: jrmora@ ut.edu.co

Artículo recibido: 24 de octubre de 2014.

Aprobación definitiva: 24 de enero de 2015. 


\title{
CRISIS OF THE COLOMBIAN AGRICULTURAL SECTOR: WHAT IS THE RESPONSIBILITY OF PUBLIC POLICY?
}

\author{
By: Elizabeth Rodríguez Gloria Lucía Martínez y Jairo Mora-Delgado
}

\begin{abstract}
The agricultural sector is a strategic sector for the sovereignty of a country, because it is the natural source of food and raw materials required by the society to meet basic needs. This article aims to reflect the evolution of public policies related to agriculture. We reviewed published expert papers in the last thirty years, It is concluded that the current policy of the Colombian government has followed to the letter???? The dictates of the Washington Consensus and he have ruled nothing suitable policies for national agricultural development.
\end{abstract}

Keywords: policy, neoliberalism, protectionism, agriculture, food safety

JEL Classification: Q. 180 


\section{Introducción}

El sector agropecuario resulta estratégico para la soberanía nacional porque es la fuente natural de alimentos y materias primas requeridas por la sociedad para garantizar satisfactores de necesidades básicas. Si bien, dentro de la doctrina de la fisiocracia, la tierra era el factor de la producción generador de riqueza, la cual constituía una premisa importante en la generación del desarrollo hasta el tercer tercio del pasado siglo, otras doctrinas fueron remplazando dichos postulados para dar paso a aquellas que realzan la primacía del capital y el mercado como panaceas del crecimiento económico. En ese proceso, la tierra y sus frutos comienzan a ser desdeñados por los tomadores de decisiones políticas. En el caso colombiano, sectores productores de preciados elementos para la seguridad alimentaria (carne, leche y cereales como el maíz y el arroz) llegaron a un estado de postración en los últimos 20 años, crisis que también afectó a commodities de importancia tradicional en la generación de divisas, como el café (Suarez, 2014).

Por otra parte, dentro del marco de modernización del Estado, bajo sistemas económicos y políticos complejos, uno de los mayores cuestionamientos en el debate público, tiene que ver con la capacidad de los gobiernos para actuar con suficiente autonomía ante la presión de aquellos grupos con intereses particulares y responder de manera eficiente a los múltiples requerimientos de los gobernados.

Desde la aparición misma del Estado, las preocupaciones sobre las actividades desarrolladas por éste, ocupan con prioridad las reflexiones que intentan definir o por lo menos acercarse a la mejor manera de actuar de quienes lo conducen. La diferencia hoy, radica en las incontenibles presiones que deben soportar los Estados en cuanto a las nuevas y cambiantes dinámicas de una sociedad mundial, en la que conceptos como transnacionalización, globalización e integración ya no son una posibilidad sino una imposición.

Bajo este esquema, ya no basta con analizar la función estatal a partir de la acción del gobierno como hecho inherente a su existencia, sino que se debe establecer técnicamente cuál es el rol que éste debe desempeñar y el estilo de gestión más apropiado para enfrentar las situaciones de crisis propias de una aldea global con enormes desequilibrios. Los gobiernos, a lo largo del tiempo, han hecho uso de herramientas diseñadas específicamente para alcanzar sus propósitos como gestores de cambio, una de estas corresponde a las políticas públicas, las cuales se han consolidado como parte esencial del quehacer político y técnico-práctico de la gestión pública.

La intención principal de este análisis es hacer una reflexión de la evolución de las políticas públicas relacionadas con el sector agropecuario. Para ello se revisaron documentos publicados en los últimos treinta años, lapso en el cual los hechos demuestran una degradación de la política sectorial y una exposición progresiva del sector agropecuario a las medidas de la política macroeconómica, muchas de las cuales han representado una amenaza para el sector. 


\section{El papel de las políticas públicas en el desarrollo sectorial}

A partir de 1950 la producción literaria sobre políticas públicas ha sido significativa y ha generado múltiples definiciones y controvertidos debates sobre la razón de ser de la misma. Entre otros, los trabajos de Laswell (1951), Lindblom (1992), Dror (1971), Aguilar (1996), Kingdon (1995), Meny y Thoening (1992), Valencia y Álvarez (2008) y Velásquez (2009) pueden resaltarse como los pioneros en el desbroce del camino para lograr un estatuto teórico de la esencia de la política pública. Con base en tales trabajos se han intentado múltiples definiciones que para efectos del presente análisis y sin pretender creer que sólo una acepción es la correcta, limitándose la posibilidad de generar definiciones interesantes desde diferentes perspectivas, entenderemos como política pública: la construcción social liderada de manera anticipada y racional por un gobierno, que se materializa a través de planes, programas y proyectos globales o sectoriales, donde el fin último es generar un producto, un resultado, o en términos sociales, un beneficio que mitigue o corrija una situación reconocida como problemática y que además como valor agregado contribuya a mejorar las condiciones para que dicha situación no conlleve a una inminente crisis.

Concebir la política pública como una construcción social, sugiere necesariamente que su formulación, implementación y evaluación se debe realizar bajo niveles de participación considerable de los diferentes grupos de interés, para una solución efectiva de las problemáticas vividas por éstos. No obstante, esta idea pareciera utópica en cuanto que en países en desarrollo como Colombia, las políticas públicas han sido estimuladas por intereses poco virtuosos, orientados a lograr casi siempre una legitimación electoral del gobierno de turno o el beneficio de sectores considerados como poderosos.

Tradicionalmente en países como Colombia, la construcción de las políticas públicas se ha efectuado bajo esquemas verticales y tecnocráticos que el Estado proyecta equivocadamente a través de las decisiones de instituciones y corporaciones públicas ${ }^{4}$. Ante el centralismo tecnocrático, lo conveniente sería la formulación de políticas públicas que trasciendan la acción gubernamental, en la medida que lo "público" se refiere a aquellas políticas que devienen en interés general y su decisión resulta del diálogo, la transparencia y la accesibilidad en sus acuerdos; se entiende que en un estado social de derecho la financiación procede de las arcas del Estado (Aguilar, 1996) o al menos se debería estimular la creación de incentivos que promuevan la eficiencia del uso de los recursos públicos (Wiesner, 1980; Wiesner, 1982)

Esta situación conflictiva que ignora el carácter público de las políticas públicas, sugiere una reflexión cuidadosa sobre qué tan participativas, convenientes y efectivas han sido las políticas públicas en Colombia y cuál ha sido su aporte en la resolución de las situaciones problemáticas. En este caso, el análisis debe partir del papel que desempeñan las políticas públicas, a través de la Política Sectorial, bajo el actual esquema económico y político en el que se mueven los sectores y

4. Un ejemplo clásico sería el fracaso de la Ley 100 de Salud en Colombia, donde los grupos afectados constituyen el foco de análisis pero sin involucrárseles como parte fundamental de la solución. 
subsectores del país. El caso que en este manuscrito nos ocupa y siguiendo la tipología propuesta por Vargas (1999) en función del área sustantiva que atenderá la política es el sector agropecuario.

\section{Importancia de la política sectorial}

En los años cincuenta, sesenta y setenta, la sustitución de importaciones fue la filosofía predominante en América Latina, en la que en el siglo XX Colombia tuvo un desarrollo relativamente exitoso, más para la economía en su conjunto que para la agricultura, con base a incentivos similares en términos de protección y subsidios. Con este apoyo, la agricultura crece bien entre 1930 y 1980, pero decae entre 1980 y 2000 (Kalmanovitz y López, 2005). Pese a algunos logros en cuanto a crecimiento y generación de una base industrial no despreciable, este modelo entró en crisis a principios de los años ochenta, cuando el continente enfrentó graves crisis de balanzas de pago que caracterizaron la llamada "década perdida".

En las postrimerías de los ochenta y principios de los noventa se propone el llamado "Consenso de Washington" que limita el papel del Estado y crea las condiciones para el funcionamiento de mercados liberados. En su lugar se promueve la iniciativa privada a todas las áreas de la actividad económica y en especial se adopta el monetarismo ${ }^{\mathbf{5}}$, doctrina político económico que desde los años 50 había hecho oposición a las políticas de intervención económica de inspiración keynesiana. En esencia, los neoliberales se oponen a las políticas sectoriales particulares, porque consideran que la forma de lograr la eficiencia y maximizar el crecimiento es mediante el establecimiento de un escenario macroeconómico estable y uniforme, cuyas reglas sean válidas para todo el mundo, sin crear preferencias sectoriales, discriminaciones ni distorsiones (Kay, 2004: 16).

Tales transformaciones constituirían en el futuro el núcleo de las políticas de gobierno que girarían en torno a un mercado globalizado, donde los sectores dependen de las ventajas comparativas con las que cuenten y las competitivas que desarrollen ${ }^{\mathbf{6}}$, además de la formulación y ejecución de políticas públicas que debían apuntar al mejoramiento continuo de su productividad, desde una perspectiva moderna y de largo plazo que contemple factores determinantes para la sostenibilidad, a través de acciones que le apunten a la innovación, la formación acertada del talento humano y el respeto al medio ambiente. Naturalmente, el modelo teórico implicaba la inversión en esfuerzos para la creación de políticas públicas generadoras de múltiples impactos positivos, para crear un ambiente propicio a las economías nacionales, esto como basamento para lanzarse al juego de las leyes de mercado, donde el papel del Estado no interfiriera en los procesos de desarrollo macroeconómico.

5. El monetarismo es la rama o vertiente del pensamiento económico neoliberal desarrollado en la Escuela de Chicago que se ocupa de los efectos del dinero sobre la economía en general y se basa en postulados de la escuela neoclásica en política económica.

6. El neoliberalismo postula básicamente que el mercado, en condiciones de competencia e información perfectas, es eficiente en la asignación de recursos productivos entre varios usos y los hace fluir hacia donde están las ventajas comparativas del país y del agente económico, siendo la iniciativa privada el agente central de cambio (CEPAL, 1999). 
En un sentido práctico, esto se materializaba en políticas de gobierno y, de acuerdo con la estructura organizativa de los países latinoamericanos, las políticas públicas sectoriales serían diseñadas, implementadas y evaluadas en ministerios, direcciones, secretarías, o demás entidades perfiladas para la planeación y desarrollo de un sector en particular. En este sentido Pérez (2004) sugiere unas políticas sectoriales como el centro de las estrategias de especialización o diversificación propuestas por la CEPAL como caminos necesarios para avanzar en el desarrollo productivo. Con base en ello, podría inferirse que las políticas sectoriales constituyen una vía de intervención del Estado para regular las imperfecciones derivadas de actuaciones perversas de las fuerzas del mercado.

En el caso de Colombia (2005) Rocha al analizar los Planes de Desarrollo infiere que las políticas orientadas a la conformación de sectores líderes han existido a lo largo de las últimas cuatro décadas. Durante las décadas de los sesenta, setenta y ochenta, el foco estuvo mayormente en la formulación de políticas verticales hacia sectores como el agropecuario, la construcción y el turismo, mientras que a finales de la década de los ochenta y noventa, se enfatizó más en políticas de carácter horizontal. No obstante, es notorio que en Colombia, como en los demás países latinoamericanos, se implementaron un mayor número de políticas que desencadenaron una mayor complejidad burocrática, a un mayor costo fiscal, con una relación menos transparente entre políticas y resultados. Así, se advierte una significativa reducción del peso de las políticas sectoriales en la asignación del presupuesto público colombiano, lo cual indica que las políticas sectoriales han ido perdiendo relevancia dentro de la agenda de políticas públicas, lo cual evidencia la obediencia de los gobiernos de turno a los designios del Consenso de Washington. Esto devela que en Colombia los cambios en política derivan de las agendas de instancias internacionales, y en particular de las que otorgan financiamiento, o que la situación interna sea tan crítica como para que el gobierno tenga la voluntad y la capacidad de realizar cambios (Roth, 2002; Gómez, 2008).

\section{La política agraria en Colombia}

A partir de mediados del siglo XX América Latina adoptó un modelo de desarrollo que priorizaba el sector industrial, concibiendo al Estado como actor principal en la distribución de los recursos, especialmente los provenientes del sector primario como complemento para el desarrollo industrial (Romero, 2012). El modelo, asociaba la ruralidad con esquemas pre-modernos y atrasados del desarrollo, por lo cual se promovían visiones de largo plazo que asociaran el desarrollo con lo urbano e industrial.

Simultáneamente, entre los años cincuenta y ochenta hubo políticas sectoriales promotoras de la agricultura empresarial, tendientes a amortiguar el efecto de otras políticas con orientación industrial y urbana. Es una época en la que muchas políticas agrarias (p.ej. subsidios a los insumos, al agua, al crédito y a las tarifas de diversos servicios) se diseñaron para fomentar el desarrollo de la agricultura. No obstante, ya a finales del siglo pasado algunos autores habían sentenciado que el conjunto de estas medidas de política tendría un efecto desfavorable para el sector agropecuario (Dirven, 1999; Krueger et al, 1991), en la medida que estas no 
estuvieron al alcance de la mayoría de productores rurales, sino al servicio de una élite con capacidad de aplicar el paquete tecnológico de la revolución verde para el cual se diseñaron las medidas (Holt-Giménez, 2007). Además, las políticas macroeconómicas, tuvieron un fuerte impacto negativo que no logró ser compensado por las políticas sectoriales a favor del sector agrario.

Cabe aclarar que los impactos negativos posiblemente se debieron a la naturaleza heterogénea y a los desequilibrios del sector que propiciaron inequidades en el beneficio para unos pocos privilegiados, derivado del subsidio al uso de insumos, al agua y al crédito y a las tarifas de diversos servicios usados, que eran generalmente medianos y grandes productores empresariales (Gómez, 1994), pero limitando el acceso a los mismos a los pequeños productores. Esto acompañado de manejos poco ortodoxos, propios de actuaciones clientelistas, deslegitimaron una política sectorial que bien orientada hubiese podido ser mejor aprovechada para el desarrollo del sector. Lo sobresaliente es que en una perspectiva económica, el modelo de desarrollo para el sector rural en términos del Informe Nacional de Desarrollo Humano (PNUD, 2011) se demarca en dos momentos: un momento proteccionista que operó hasta finales de los años 1980, y un momento de apertura económica que se abre espacio después de 1990. Así, los cambios en la economía agraria y en los procesos económicos, sociales y políticos del país se entienden en el ámbito del funcionamiento de esos referentes para la operación del mercado y el Estado (Jaramillo, 2002; Jaramillo et al., 1991).

Ya desde los años noventa Dirven (1999) avizoraba las tendencias que hoy se verifican con los hechos. El análisis de este autor constituía un vaticinio de las medidas de política económica que en los 15 años siguientes han constituido las agendas de las políticas públicas: procesos de liberalización de los mercados y apertura al exterior; descentralización; nuevas instancias de consulta y participación; incentivos para la concertación entre distintos agentes; nuevas alianzas público-privadas; transferencia de funciones desde el Estado a agentes privados $\mathrm{u}$ organizaciones no gubernamentales y fomento de políticas transversales en lugar de políticas sectoriales. Cambios profundos que corresponden a la emergencia de un nuevo modelo de desarrollo, caracterizado por la menor intervención del Estado y su respectiva reestructuración y políticas de ajuste estructural aplicadas al medio rural, simultáneo al desarrollo de transnacionales agroalimentarias a escala global. Tales factores han incidido en la reconfiguración de la sociedad rural latinoamericana, expresada en las tendencias señaladas (Romero, 2012).

En la institucionalidad colombiana, tales transformaciones quedarían consagradas con la aparente legitimación de la sociedad en la Asamblea Nacional Constituyente que emitiría una nueva carta magna, que preparaba el andamiaje jurídico político de un nuevo orden económico acorde con las tendencias globales aperturistas, elevándolas a políticas de Estado.

Los años 90 representaron una década crucial para las reformas políticas y económicas que rediseñaron el Estado y la política sectorial. Perry (2000) menciona que desde el segundo semestre de 1990 hasta finales de 1992 se pusieron en marcha la mayoría de las reformas iniciales, orientadas por la liberación comercial y desregulación de la economía; entre 1993 y 1995 se cumplió una etapa de formu- 
lación de una nueva política sectorial, o de una segunda generación de reformas, y desde 1995 hasta finales del siglo XX se vivió un período de ejecución de parte de lo diseñado.

Del 2000 en adelante, en general, se ha implementado lo diseñado en el marco de una concepción neoliberal del Estado, tendiendo cada vez más a una reducción de sus actividades y la delegación de responsabilidades al sector privado que por naturaleza se rige por la lógica del mercado. Sin embargo, en el sector agropecuario son evidentes los vacíos de los que no se ha hecho cargo aún cabalmente el sector privado y lo más seguro es que no los asumirá sin compensación en áreas poco rentables. De hecho, el ahondamiento de estos vacíos era de esperarse en virtud de que una economía de mercado limitada en su desempeño por imperfecciones en la información, en los mercados de capital y en la competencia (Stiglitz, 1994) lo más probable es que desemboque en los llamados "fracasos de mercado" los cuales justifican la intervención estatal para regular la asignación de recursos y corregir los desvíos. Greenwald y Stiglitz lo habían expresado hace 30 años al sentenciar que una economía de mercado con estas imperfecciones viola los principios de la Economía del Bienestar al no dar espacio para mejorar a algunas personas sin desmejorar a otras, por lo cual se requiere entonces de la intervención del Estado si se desea un incremento en el bienestar de la población, ante la imposibilidad de conseguir este resultado a través del libre juego de la oferta y la demanda (Greenwald y Stiglitz, 1986). Aun defensores del mercado en Colombia, como Eduardo Wiesner, reconocen que el mercado no es intrínsecamente bueno sino que requiere un orden institucional que permita conjugar el bienestar de los individuos con el bienestar colectivo (González, 2006: 18)

\section{La ausencia del Estado en el sector primario ha tenido efectos adversos}

En especial para los pequeños y medianos productores, manejadores de una agricultura de pocos recursos y tierras marginales, quienes vieron poco a poco limitarse el acceso al crédito, a la asistencia técnica agrícola, a los mercados y a los mecanismos de compra de sus cosechas, centros de acopio y a la generación de una ciencia y tecnología apropiada.

Tal vacío dejado por el Estado fue asumido por los gremios de la producción y algunas organizaciones no gubernamentales, especialmente orientadas a atender la producción de pequeña escala. Sin embargo, la heterogeneidad y dispersión de estas últimas han enfrentado dificultades de coordinación, seguimiento y retroalimentación aún mayores que cuando el desarrollo rural estaba a cargo de los servicios de extensión del Estado.

La actual política nacional, establecida en el Plan Nacional de Desarrollo 2010 2014, teóricamente tiene como propósito aumentar la competitividad de la economía y la productividad de las empresas y considera el sector agropecuario como uno de los cinco sectores con alto potencial de crecimiento que impulsarán el desarrollo económico del país. Todo esto enmarcado en el Plan Nacional de Desarrollo (Ley 1450 de 2011) en el que se indica un plan de inversiones que impulse las "Locomotoras" por iniciativa privada, señalando el orden de prioridades que ubica a 
la actividad minero-energética como una prioridad, seguida por la agroindustria (Pérez, 2012) y en concordancia con intereses transnacionales.

Lo que preocupa es que las políticas pensadas para lograr estos objetivos constituyen quimeras o generan graves lesiones para el sector agropecuario y por ende para la economía y soberanía nacional. Veamos algunas evidencias y que de hecho, estas muestran que los lineamientos de política pública para el sector rural en nada abogan por su dinamización y por el contrario cada día lo dejan en un estado de postración.

\section{Ilusiones para el sector agropecuario}

Si bien el gobierno colombiano posee una estructura de intervención en los asuntos sectoriales, tal intento no pasa de ser un artificio dados los hechos que develan la prevalencia de la política macroeconómica. Así, en Colombia, el Ministerio de Agricultura y Desarrollo Rural, cuenta con una Dirección de Política Sectorial, la cual se encarga de formular la política del sector agropecuario, pesquero y de desarrollo rural. No obstante, los instrumentos que viabilizarían sólidos lineamientos de política en el marco de una verdadera planificación estratégica, se toman como accesorios y no como verdaderas herramientas que deberían apalancar la política del sector. Muestra de ello es la deficiencia en los sistemas de información, el desmonte de los servicios de asistencia técnica y extensión rural y la precariedad en el apoyo a ciencia y tecnología en materia agropecuaria.

- Precariedad en los servicios de información: La encuesta semestral de área y producción agropecuaria denominada ENA y las evaluaciones de consenso denominadas Evaluaciones Agropecuarias EVA, han sido procedimientos adoptados por el Ministerio de Agricultura desde la década de los setenta para obtener información sectorial a nivel municipal sobre área, producción y rendimiento de los principales cultivos e información pecuaria y piscícola. Estas se realizan con el apoyo de supuestos expertos y entidades locales conocedores de la actividad agropecuaria y de las Secretarías de Agricultura. Las EVA se basan en la metodología de los consensos regionales y departamentales, instancias de discusión y análisis de la información recopilada fundamentalmente por los técnicos de la Unidades Municipales de Asistencia Técnica (UMATA) (Ministerio de Agricultura y Desarrollo Rural, 2012). Si bien, estos mecanismos, representan el insumo básico para la formulación de la política del sector, tal proceder para el diseño de política no es el más apropiado y no puede sustituir un verdadero censo agropecuario.

Ya era hora que después de 44 años en el país se realice el tercer Censo Nacional Agropecuario con el fin de hacer un estudio exhaustivo y riguroso del sector rural. Esto permitirá conocer la realidad del sector agropecuario para que la toma de decisiones y la formulación de políticas públicas sean coherentes con la realidad del sector. Según Gossain (2014), no es concebible que estas últimas cuatro décadas se hayan tomado decisiones cruciales para el país y su economía sin saber a ciencia cierta lo que poseemos. Ya resultados preliminares del Censo develan la persistencia de una distribución inequitativa de tierra caracterizada por una alta concentración en pocas manos, especialmente en las 
sabanas de la costa Caribe y en la Orinoquia colombiana y una predominancia del fraccionamiento de la propiedad en Antioquia, Cundinamarca, Boyacá y los Santanderes, donde se concentra más del 50\% de los predios.

Ya lo había dicho Perfetti (2012) que la caracterización del inventario agropecuario del país permitirá identificar la estructura productiva y el uso de la tierra y se podrán establecer estructuras de tecnificación productivas, el acceso a asistencia técnica, entre otros datos fundamentales para establecer una radiografía de la realidad del campo colombiano. Es de suponer, que para determinar las fortalezas y las debilidades de la base productiva de un país, se deben tener actualizados las estadísticas generales, en este caso, el agropecuario general ${ }^{7}$. Los habitantes rurales tendrán que esperar que este nuevo intento no sea otra de las quimeras mal logradas.

- El cambio tecnológico: otra quimera. Una condición fundamental en el sector agropecuario para que esté preparado para la competencia, es la necesidad de ponerse al día en avances tecnológicos que les permita a los agricultores un nivel aceptable de modernización y eficiencia. Naturalmente, como lo demuestra la historia, en la segunda posguerra en el mundo moderno desarrollado, esa responsabilidad fue liderada por el Estado. Sin embargo, la década de los 90 fue testigo de una tendencia mundial hacia un papel más reducido del sector público y una reducción en la intervención gubernamental en la agricultura manifiesta en una restricción del gasto público. Además, se comienza a perder el apoyo de agencias internacionales de ayuda, paralelamente al debate conceptual que sobre el alcance de la extensión comenzaba a darse, en virtud de la creciente complejidad del desarrollo agrario que exigía nuevas aproximaciones para interpretarlo (Dirven, 1999). Ya desde esa época, expertos como Trigo y Kaimowitz (1994) auguraban un efecto directo en los institutos públicos de investigación agrícola como en los servicios de extensión. Así, en tales tiempos, como ahora, las iniciativas de investigación estratégica requerirán participación directa del sector público, en virtud de que no cuentan con una aplicación comercial de corto plazo ${ }^{8}$.

Paralelamente, desde los años 70 la extensión agropecuaria comenzó a caer en un estado de postración, situación derivada de una progresiva crisis institucional de los servicios nacionales de extensión. Además, el proceso de burocratización que se evidenciaba en los servicios conllevó a que estos se convirtieran en fortines políticos e hizo que gradualmente perdieran su agilidad, autonomía y flexibilidad, dando pie a las pretensiones del Estado de reducirlos a su mínima expresión.

7. Mientras que en otros países del mundo los censos son una herramienta fundamental para generar información del universo de productores agropecuarios y de los hogares rurales que ocupan el territorio rural del país, para hacer seguimientos a diversas variables, al tiempo que permite la construcción de muestras para adelantar estudios especializados.

8. Por ejemplo, la conservación de recursos naturales, investigación agronómica diseñada para la reducción de costos y apoyo para pequeños agricultores y cosechas de productos alternativos. 
En ese proceso, la extensión agraria fue perdiendo el protagonismo en la promoción de nuevas variedades mejoradas, uso de insumos agroquímicos y uso de buenas prácticas agropecuarias, para ceder este terreno a las casas comerciales que pronto lo asumieron con los beneficios que aquella les representaba.

Así, sin el suficiente apoyo presupuestal necesario para cumplir su misión, los servicios de extensión se fueron desmontando en América Latina: en 1979 comenzó este proceso en Chile, seguido poco después por México y Bolivia (Trigo y Kaimowitz, 1994); en Colombia esta responsabilidad se descentralizó asignándola a los municipios con la creación de las Unidades Municipales de Asistencia Técnica (UMATAS) (Perry, 2000), con las obvias consecuencias derivadas de un manejo clientelista a nivel local, agravado por los reducidos presupuestos que los gobiernos municipales asignaron para la extensión. Un proceso parecido se vivió en Brasil donde se cerró la institución federal responsable de la extensión para dar paso a que cada estado determine su propia política de extensión agropecuaria.

En Colombia, la experiencia de burocratización de las Umatas, pronto dio paso a otras formas de alianzas público privadas que asumieran la asistencia técnica, así, los Centros Provinciales de Gestión Agroempresarial CPGA cundieron por el territorio nacional como instrumentos para la gestión de recursos de desarrollo agropecuario que incluían estrategias de asistencia técnica, aunque hasta la fecha no se haya evaluado.

- El desmonte de la investigación agropecuaria. En los años 90, en casi todos los Institutos Nacionales de Investigación Agropecuaria (INIA) de la región, se forma un ambiente caracterizado por la escasez relativa de fondos para la investigación, la creciente apropiación de la tecnología y el surgimiento de nuevas demandas (Dirven, 1999). Con la nueva Constitución de 1991, ideada para acondicionar el Estado colombiano a los designios del modelo económico aperturista vía privatización de las empresas estatales, se aprobó una reestructuración a fondo del Instituto Colombiano Agropecuario -ICA-, único ente de investigación agropecuaria, generando un modelo distinto de gestión estatal de la investigación agropecuaria, que buscó transferir a los particulares la responsabilidad de la generación de tecnología agropecuaria. Tal reforma implicó una reasignación de funciones en la planificación del conjunto de la actividad de generación de tecnología agropecuaria y en su distribución presupuestal, además de separar las labores de regulación, prevención y control sanitario de la de generación y transferencia de tecnología. Así, en lo subsiguiente las primeras se reservaron para el ICA y las segundas deberían ser ejecutadas por otras entidades, principalmente por corporaciones mixtas de derecho privado, de las que contempla la legislación de ciencia y tecnología mencionada, con lo que dio paso a la creación de CORPOICA como la más importante de ellas. 
Corpoica se creó a principios de 1993 como una corporación mixta de derecho privado (Perry, 2000) ${ }^{\mathbf{9}}$.

Veinte años después de dichas reformas es evidente que el desmonte del andamiaje institucional sobre el cual descansaban la generación y transferencia de tecnologías, no resolvió el problema de ineficiencia. Actualmente, el modelo Corpoica tiende a la crisis, pues el sector privado no ha asumido el papel que el modelo de privatización le asignó y la Corporación se ha visto obligada a competir de manera desleal con sus propios socios (las Universidades) en la búsqueda de los escasos recursos para la investigación que Colciencias o el Ministerio de Agricultura asignan mediante concursos ineficientes.

- ¿Desatención a las políticas transversales? El desestímulo de las políticas sectoriales daría paso a políticas públicas transversales, bajo el supuesto de que estas se convirtieran en dinamizadoras del conglomerado productivo y como estrategia de preparación de las competencias que requería la dinámica del mercado. La política educativa constituye un ejemplo claro de una política transversal que debía apoyar el modelo de desarrollo. Stitgliz ya a finales del siglo pasado advertía de la necesidad de la creación de capacidad en la fuerza laboral como un factor clave de la economía del conocimiento. Para ello, muchos países se habían enfocado a mejorar sus sistemas educativos. En el largo plazo el éxito de la economía del conocimiento requiere el desarrollo de creatividad y de altas destrezas cognitivas además de las destrezas básicas. Así, aquellos países que encuentran caminos adecuados en esta clase de creatividad tendrían más éxitos en la competencia de la economía del conocimiento. Esto se logra con un entrenamiento en ciencia y tecnología (Stitglitz, 1999). Sin embargo, los hechos ponen en evidencia que el modelo teórico dista mucho de los desempeños en materia educativa, generando grandes brechas que ponen en duda la efectividad de las políticas públicas en este sector, especialmente en un país en desarrollo como Colombia.

Una de las principales razones de fondo de la asimétrica competencia entre Colombia y los bloques de países con quienes se han suscrito TLC se debe a que el sector productivo nacional es poco intensivo en conocimiento y carece de capacidad de innovación. Carece esencialmente de doctores en ciencia y tecnología, dado el bajo número de graduados en áreas de las ciencias agrarias, con relación a los graduados en ciencias básicas y en ciencias sociales.

\section{Los retos de la política pública para resarcir acuerdos "leoninos"}

Cumplidos dos años del inicio formal del TLC entre Colombia y Estados Unidos, se comienzan a vislumbrar los fantasmas del pasado aperturista de nuestro país,

9. CORPOICA se fundó con más de cien socios entre ellos el Gobierno Nacional, a través del Ministerio de Agricultura y del ICA, la mayoría de las agremiaciones de productores agropecuarios de carácter nacional y varias de carácter regional; las principales universidades del país, tanto nacionales, como regionales, públicas y privadas; diversos centros de investigación; algunos departamentos y municipios, y varias empresas privadas. 
pues el libre comercio del siglo XXI es un lastre para un sector que nunca superó los índices abismales de atraso y como consecuencia, es sometido al vaivén de acuerdos inequitativos y asimétricos.

Barberi (2014), hace hincapié en que este acuerdo comercial caracteriza al sector agrario como perdedor; pues el gobierno colombiano ha sido permisivo y genuflexo, permitiendo a todo costo el ingreso de toneladas de productos sin aranceles, provocando una competencia desleal en la oferta de los mismos, agravado por el bajo precio de las importaciones debido a que llegan libres de tributo y subsidiadas por el gobierno estadounidense.

Es posible afirmar que la negociación del TLC con los Estados Unidos ha sido una de las manifestaciones más importantes de la política de "internacionalización" de la economía adoptada por el Gobierno Nacional hace más de 20 años (Barberi, 2014). Es inadmisible, que en pleno desarrollo de la estructura de la negociación, se evidenciara el desconocimiento de la base productiva y el mínimo grado de desarrollo del sector. De hecho, si a ese nivel, se tuviese completamente claro, analizado y estudiado a detalle la solidez de la economía del país socio (los Estados Unidos) no sería difícil detectar las asimetrías que constituyen desventajas para nuestro frágil sector agropecuario, en temprano crecimiento (Pérez y Valencia, 2011; Suarez 2013)

El holocausto del libre comercio ya tenía sus víctimas señaladas: el campo colombiano. Las consecuencias negativas comenzaron a notarse en los principales cultivos transitorios, algunas leguminosas, hortalizas y actividades pecuarias como la producción de pollo y cerdo.

Bajo estas circunstancias inequitativas en las que sobresale el principio de la "libre competencia", es de esperarse que se desestabilice la producción nacional, obligando a los pequeños y medianos productores a someter sus cultivos a precios de hambre, lo que posiblemente desencadene una disminución en el área sembrada, menor ocupación laboral, desplazamiento en busca de mejoras en el ingreso y demás situaciones sociales que atentan contra el anhelo del desarrollo.

\section{Consideraciones finales}

Las políticas sectoriales han sido desdeñadas desde la década del 80, manteniéndose descartadas del nuevo esquema de modelo económico implantado con las reformas estructurales, esto en parte, por el rol de "Estado Subsidiario" que se imponía y los continuos fracasos en la gestión pública, derivados generalmente del mal manejo que conllevó al ejercicio del clientelismo y la corrupción, lo cual deslegitimó las políticas. Con la llegada del nuevo siglo, la poca democratización de los medios de producción, la inequitativa distribución de la riqueza, el precario acceso a la educación superior, los escasos recursos para lograr transferencia de conocimiento en ciencia, tecnología e innovación y lo más grave la insuficiente participación de la sociedad en los asuntos de interés público, desvirtuaron aún más los posibles beneficios de la política sectorial.

Hoy, las políticas públicas sectoriales deben repensarse de acuerdo con las características propias del país, a fin de establecer un enfoque de desarrollo para 
el área de su competencia, y generar acciones intersectoriales, también, se debe mantener una estructura lógica con la planeación para el desarrollo, establecida para el ámbito nacional.

Por tal razón, es indispensable asegurar que los sectores mejoren sus niveles de desempeño y potencialicen sus ventajas comparativas, por ejemplo, Colombia, con una vocación tradicionalmente agropecuaria, debería implementar acciones que conlleven a incentivar su soberanía alimentaria y ganar espacios en los mercados mediante una producción diferenciada, limpia y sostenible, que cuente con los recursos necesarios para integrarse con éxito al mercado interno y global, y que consecuentemente además de aumentar los ingresos de los productores, preserve los ecosistemas y mejore la calidad de vida de la sociedad rural.

Si bien es cierto, que las políticas sectoriales deben considerar acciones inmediatas como programas y proyectos para lograr objetivos específicos, es pertinente establecer acciones dirigidas a mantener planes de largo plazo que le apunten a objetivos más generales, de modo tal que en algún momento la planeación nacional trascienda las políticas de gobierno y llegue en algunos casos a consolidar políticas de Estado.

Los documentos revisados, permiten concluir que la política actual del Gobierno colombiano no aporta nada nuevo, y lo peor, nada conveniente para el desarrollo del sector agropecuario nacional que lo prepare para la embestida que implican los tratados de libre comercio. La mayoría de ellos, con países que sí protegieron y fortalecieron su sector agropecuario hasta llevarlo a constituirse en un sector de interés estratégico nacional.

La política pública en Colombia tiene que verse conectada e integrada en todos los niveles, por ejemplo, una vez consolidada una política pública de ciencia y tecnología, con grandes recursos para que sea de calidad y al servicio de las necesidades más sentidas de la población, será la pionera de los avances tecnológicos de innovación que requiere el sector agropecuario, el cual ya no tendría que depender de los diseños foráneos, subyugándose a la experimentación improvisada con la tierra.

La política pública para el sector agropecuario debería reorientarse, para ponerla al servicio de los productores nacionales y de la soberanía alimentaria, además de propender por desarrollar la capacidad educativa y tecnológica de alto nivel, que les permita a los productores nacionales un mejor desempeño para volver eficientes sus sistemas de producción, trasformación y distribución, con lo cual mejorá la capacidad de competencia frente a sus pares internacionales. Para ello, se requiere que la política agropecuaria sea concebida como política estratégica de Estado. 


\section{REFERENCIAS}

1. AGUILAR, L F. (1996). Estudio introductorio. En: Aguilar, Luis Fernando. El estudio de las políticas públicas (pp. 5-74). Miguel Ángel Porrua. México.

2. BARBERI, F. EI TLC: dos años después, un balance: Los campesinos son los grandes perdedores. Mayo, 2014. http://www.las2orillas.co/el-tlc-dos-anos-despues-un-balance/

3. CONTEXTO GANADERO. (2012). DANE anuncia aplazamiento del Censo Agropecuario 2013. On line. http://contextoganadero.com/economia/dane-aplaza-censo-agropecuario-2013

4. DIRVEN, M. (1999). El papel de los agentes en las políticas agrícolas: intenciones y realidad. Revista de la CEPAL No. 68, 171-186.

5. DROR, Y. (1971). Design for Policy Sciences, Elsevier, New York.

6. GÓMEZ Oliver, L. (1994). El papel de la agricultura en el desarrollo de México, Chile. Organización de las Naciones Unidas para la Alimentación y la Agricultura (FAO).

7. GOSSAIN, J. (2014). El censo agropecuario descubre una Colombia inesperada. El Tiempo. Lunes 27 de octubre. 1 p.

8. GÓMEZ MI. (2008). Reseña de políticas públicas: formulación, implementación y evaluación de AndréNoël Roth Deubel Revista Opera. №. 8. 202-204 p.

9. GONZALES, JL. (2006). Los aportes de Eduardo Wiesner al pensamiento económico colombiano. Rev. econ.inst. v. 8 n. 14.

10. GREENWALD, B. \& J.E. STIGLITZ. 1986. Externalities in economies with imperfect information and incomplete markets. Quarterly Journal of Economics 101: 229-264.

11. HOLT-GIMÉNEZ, E., ALTIERI, M y ROSSET, P. (2007). Rockefeller y Bill Gates NO resolverán los problemas de pobreza y hambre en África. Ecoportal.net.

12. JARAMILLO, Carlos Felipe (2002). Crisis y transformación de la agricultura colombiana. Banco de la República, Fondo de Cultura Económica. Bogotá.

13. JARAMILLO, Carlos Felipe; PERFETTI, Juan José; RAMÍREZ, Jorge (1991). Modelos de desarrollo, sector agropecuario y desarrollo rural: hacia un nuevo paradigma. En: Desarrollo rural y apertura económica (primera edición). Seminario Internacional DRI, Ministerio de Agricultura, IICA. Bogotá.

14. KAY, C. (2004). Enfoques sobre el Desarrollo Rural en América Latina y Europa desde mediados del siglo veinte. Institute of Social Studies, La Haya, Holanda. Consultado en Internet en abril de 2013. http:// www.ruta.org:8180/xmlui/bitstream/handle/123456789/600/RN138.pdf?sequence $=1$

15. KALMANOVITZ, S. y LÓPEZ, E. (2005). Aspectos de la agricultura colombiana en el siglo XX. http://www. salomonkalmanovitz.com/Ensayos/Aspectos\%20de\%20la\%20agricultura\%20colombiana\%20en $\% 20$ el\%20siglo\%20XX.pdf

16. KINGDON J.W. (1995). Agendas, alternatives and public policies (Longman Classics Edition) (2nd Edition). USA. 245p.

17. KRUEGER, A.O. (1995). Policy lessons from development experience since the Second World War, en J. Behrman y T. N. Srinivasan (eds.), Handbook of Development Economics, Vol. IIIB, North-Holland Publishing Company, Amsterdam. 
18. LASSWELL, Harold D. (1951b). The policy orientation. In The policy sciences: recent developments in scope and method, eds. Daniel Lerner and Harold D. Lasswell. Stanford University Press. Stanford.

19. LINDBLOM, C. (1992) Inquiry and change. New Haven, CT, Yale University Press, p. 330

20. MENY, Y. y THOENING. JC (1992). Las políticas públicas, Ariel, Barcelona.

21. NAGEL, JC. (2005) Un marco para la formulación de políticas sectoriales en la región andina. En: Castilla, LM; Manzano, O y Nagel, J (Eds,). Políticas sectoriales en la región andina. Lecciones y propuestas. CAF, Caracas. 387 p.

22. PERES, W. (2004). Políticas sectoriales y de desarrollo de clusters en América Latina y el Caribe. CEPAL. Santiago de Chile. $35 \mathrm{p}$.

23. PÉREZ, M. (2012). Políticas agrarias en Colombia: Una mirada desde el desarrollo legislativo. En: Cuadernos de Semillas. No. 2. Bogotá, 8-14 p.

24. PÉREZ, TH y Valencia MA (2011). Comercio exterior y atraso en la producción el TLC entre Colombia y la Unión Europea. RECALCA, Bogotá. 65 p.

25. PERFETTI, JJ. (2012). ¿Para qué un Censo Agropecuario? En El Colombiano. Publicado el 12 de agosto de 2011.

26. PERRY, S. (2000). Impacto de las reformas estructurales en la agricultura colombiana. CEPAL-ECLAC. Santiago de Chile. 41 p.

27. PNUD. (2011). Colombia rural. Razones para la esperanza. Informe Nacional de Desarrollo Humano 2011. PNUD, Bogotá.

28. ROTH, AN. (2002). Políticas públicas. Formulación, implementación y evaluación. Ediciones Aurora, Bogotá. 220 p.

29. ROMERO, J. (2012). Lo rural y la ruralidad en América Latina: categorías conceptuales en debate. Psicoperspectivas Vol. 11, № 1. 8-31p.

30. SUÁREZ, A. (2013). Primeras lecciones del TLC con EE.UU. El Espectador. Consultado en: http://www. elespectador.com/opinion/primeras-lecciones-del-tlc-eeuu-columna-422024

31. SUÁREZ, A. 2014. Los TLCy la crisis del sector rural tocó fondo. Análisis de la actual problemática agraria. Entrevista a Aurelio Suárez Montoya. Revista Semillas. № 53/54.

32. SUE (2012). Universidades públicas claman al Congreso para que revise a su favor el presupuesto 2013. Observatorio de la Universidad Colombiana.

33. STIGLITZ, J.E. (1994). Whither Socialism? MIT Press. London.

34. TRIGO, EY KAIMOWTIZ, D. (1994). Investigación agrícola y transferencia de tecnología en América latina en los años noventa. Cuadernos de Ciencia \& Tecnología, Brasilia, v.11, n.1/3, p.99-126.

35. VARGAS, A. (1999) Notas sobre el Estado y las políticas públicas, Bogotá, Almudena Editores, p. 66.

36. VELÁSQUEZ, R.G. (2009) Hacia una nueva definición del concepto "Política Pública", Desafíos, Bogotá (Colombia), (20): 149-187, semestre I de 2009.

37. VALENCIA, G y ÁLVAREZ, Y. (2008). La ciencia política y las políticas públicas: notas para una reconstrucción histórica de su relación. Estudios Políticos, 33, Instituto de Estudios Políticos, Universidad de Antioquia, 93-121.

38. WIESNER, E. (1980). El financiamiento de la inversión pública, Revista del Centro de Estudios Colombianos 25, pp. 4-10.

39. WIESNER, E. 1982. El origen político del desequilibrio fiscal, déficit fiscal en Colombia. Bogotá, Foro Económico Nacional, CGR y FESCOL, pp. 125-144. 